\title{
Corela
}

Cognition, représentation, langage

HS-17 | 2015

Regards sur l'interdisciplinarité en science du langage

\section{Regards sur l'interdisciplinarité en sciences du langage}

Actes des $15^{\text {èmes }}$ Rencontres Jeunes Chercheurs de l'Université Paris 3 Sorbonne Nouvelle

\section{Aurélia Elalouf et Marine Le Mené}

\section{(2) OpenEdition}

\section{Journals}

Édition électronique

URL : http://journals.openedition.org/corela/3745

DOI : $10.4000 /$ corela.3745

ISSN : 1638-573X

Éditeur

Cercle linguistique du Centre et de l'Ouest - CerLICO

Référence électronique

Aurélia Elalouf et Marine Le Mené, "Regards sur l'interdisciplinarité en sciences du langage », Corela

[En ligne], HS-17 | 2015, mis en ligne le 30 mai 2015, consulté le 20 avril 2019. URL : http://

journals.openedition.org/corela/3745; DOI : 10.4000/corela.3745

Ce document a été généré automatiquement le 20 avril 2019.

\section{(c) (i) (3) (2)}

Corela - cognition, représentation, langage est mis à disposition selon les termes de la licence Creative Commons Attribution - Pas d'Utilisation Commerciale - Partage dans les Mêmes Conditions 4.0 International. 


\title{
Regards sur l'interdisciplinarité en sciences du langage
}

Actes des $15^{\text {èmes }}$ Rencontres Jeunes Chercheurs de l'Université Paris 3 Sorbonne Nouvelle

\author{
Aurélia Elalouf et Marine Le Mené
}

\section{Éditorial}

1 Ce numéro thématique de la revue Cognition REprésentation LAngage regroupe une sélection d'articles issus de communications présentées en 2012 aux $15^{\text {èmes }}$ Rencontres Jeunes Chercheurs organisées par les doctorants et doctorantes de l'École Doctorale 268 «Langage et langues : description, théorisation, transmission » de l'Université Sorbonne Nouvelle - Paris 3, et qui se sont tenues au département de Didactique du Français Langue Étrangère (DFLE) de la Sorbonne Nouvelle les 15 et 16 juin 2012.

2 Les travaux présentés pendant ces journées ont permis de poursuivre les discussions menées l'année précédente sur l'éclatement actuel des sciences du langage. Alors que les $14^{\text {èmes }}$ Rencontres Jeunes Chercheurs envisageaient le champ des sciences du langage de l'intérieur, s'interrogeant sur l'existence et la confrontation de pratiques de recherche très diverses en son $\operatorname{sein}^{1}$, les $15^{\text {èmes }}$ Rencontres Jeunes Chercheurs se sont intéressées aux rapports que les sciences du langage entretiennent avec les autres domaines du savoir. L'objectif de ces journées était de réfléchir à la manière dont les jeunes chercheurs en sciences du langage conçoivent la possibilité de faire dialoguer leur champ disciplinaire avec l'extérieur et dont ils exposent et justifient leur démarche de recherche lorsque celle-ci se caractérise par le recours à des outils, méthodes ou concepts issus d'autres disciplines.

3 Il va de soi qu'une telle réflexion ne peut être menée sans s'interroger sur la notion de « discipline " elle-même, sur les délimitations à la fois interne ("sous-discipline», " courant disciplinaire ») et externe des disciplines, ainsi que sur les modes d'interactions possibles entre ces dernières. Il importe ainsi de considérer dans quelle mesure, d'un point de vue tant épistémologique que socio-historique, ce qu'on appelle les « sciences du 
langage $"^{2}$ constitue une discipline en soi ou un ensemble de disciplines connexes. Il importe également d'identifier quelles sont les disciplines avec lesquelles les sciences du langage entretiennent des relations privilégiées (on peut penser notamment à la psychologie et à la sociologie) et de déterminer à quel type de pratique de recherche (pluri-, inter- ou transdisciplinaire ${ }^{3}$ ) ces relations sont susceptibles de donner lieu. Il importe enfin de se demander dans quelle mesure le linguiste est apte à faire à lui tout seul une recherche se réclamant de l'interdisciplinarité, et si, dans ce cas, on peut toujours le considérer comme un linguiste.

Les contributions réunies ici cherchent à montrer quel peut être l'intérêt d'une démarche interdisciplinaire pour l'étude de certains phénomènes langagiers et constituent autant d'exemples de confrontation des concepts, outils et résultats d'analyse des sciences du langage avec ceux d'autres disciplines. Gilles Andrianne montre comment les analyses de la linguistique reconstructive, étant donné leur caractère abstrait et hypothétique, doivent recevoir confirmation des analyses issues d'autres domaines disciplinaires. Son argumentation porte sur le terme tóxon ( «arc »), dont le statut linguistique est remis en question à la lumière d'une interprétation nouvelle des représentations historiques, littéraires et picturales de l'arc en Grèce antique. Thierry Geoffre examine les apports possibles de la didactique des sciences à la didactique de l'orthographe. Il argumente en faveur de l'utilisation de dispositifs expérimentaux de «dépassement des représentations » inspirés de la didactique des sciences pour amener les élèves à porter un regard métalinguistique sur la langue. Caroline Panis met en évidence les limites de l'analyse textuelle pour l'analyse dialogique (interdiscursive et interlocutive) des discours oraux. Elle montre que la prise en compte des données ethnographiques, dans une perspective anthropologique, est essentielle pour étudier les discours ethnicistes qui circulent dans l'espace public burkinabè et éclairer ses données langagières. Les autres articles portent sur l'analyse des représentations construites en discours, analyse rendue possible par l'emprunt à la psychologie de la notion de « représentation sociale ». Daniela Nienkötter Sardà travaille sur les représentations de la philosophie dans un corpus de manuels français et brésiliens. Camila Ribeiro s'intéresse à l'évolution des stéréotypes sur le Brésil dans les discours à vocation documentaire ou touristique. Enfin, Pascale Asmar traite des représentations du Hezbollah dans les quotidiens libanais, français et américains entre 2010 et 2011. Elle défend en particulier la nécessité de mobiliser tant les outils de la linguistique que les connaissances apportées par l'histoire, les sciences politiques et les sciences de l'information et de la communication pour comprendre comment la presse écrite fabrique et véhicule les représentations.

\section{Sommaire}

- ANDRIANNE Gilles, « Regard interdisciplinaire sur le statut de l'arc en Grèce antique »

- ASMAR Pascale, «Quel (dé)cloisonnement disciplinaire pour l'étude des représentations du Hezbollah dans la presse écrite?»

- GEOFFRE Thierry, «De la didactique des sciences à la didactique de l'orthographe : interdisciplinarité et perspectives d'exploitation de protocoles »

- NIENKÖTTER SARDÀ Daniela, «Comparaison des discours de manuels scolaires de philosophie français et brésiliens selon l'approche interdisciplinaire de l'analyse du discours »

- PANIS Caroline, «Ces discours qui ne vont pas de soi. L'apport du dialogisme dans l'analyse de discours ethnicistes au Burkina Faso » 
- RIBEIRo Camila, "Stéréotypes: images de soi ou images de l'autre? Analyse de deux exemples brésiliens »

\section{NOTES}

1. Se reporter au numéro thématique de la revue CORELA intitulé «Diversité des pratiques de recherche en sciences du langage ».

2. Cette locution tend aujourd'hui à se substituer au terme " linguistique ». Un tel changement de désignation n'est évidemment pas anodin et nous rappelle qu'identité et frontières disciplinaires sont en perpétuelle (re)construction. Sur ces questions, voir par exemple Chiss, Jean-Louis \& Puech Christian (1999), Le langage et ses disciplines, $x i x^{e}-x x^{e}$ siècles, Bruxelles : Duculot.

3. Sur ces distinctions, voir par exemple Charaudeau, Patrick (2010) "Pour une interdisciplinarité "focalisée" dans les sciences humaines et sociales" [en ligne], Questions de Communication, 17, URL : http://www.patrick-charaudeau.com/Pour-une-interdisciplinarite.html, pour qui : 1) est « pluridisciplinaire » une démarche de recherche dans laquelle chaque discipline conserve son autonomie, ses méthodes et ses outils; il s'agit «d'une juxtaposition de points de vue qui apportent chacun une connaissance particulière sur le phénomène étudié »;2) est « interdisciplinaire» une approche qui s'efforce de faire dialoguer plusieurs disciplines sur un même thème, cherchant ainsi à "établir de véritables connexions entre concepts, outils d'analyse et modes d'interprétation »; 3) est «transdisciplinaire » une démarche qui transcende les disciplines, réalisant une « intégration des savoirs [...] de telle sorte qu'émerge un discours sui generis construisant son propre lieu de pensée ».

\section{AUTEURS}

\section{AURÉLIA ELALOUF}

Université Paris 3 - Sorbonne Nouvelle

\section{MARINE LE MENÉ}

Université Paris 3 - Sorbonne Nouvelle 